\title{
Efficient Auger scattering in Landau-quantized graphene
}

\author{
F. Wendler*a, H. Funk ${ }^{a}$, M. Mittendorff ${ }^{b}$, S. Winnerl ${ }^{c}$, M. Helm $^{c}, d$, A. Knorr ${ }^{a}$ and E. Malic ${ }^{e}$ \\ ${ }^{a}$ Institute of Theoretical Physics, Technical University Berlin, Berlin, Germany; \\ ${ }^{b}$ Institute for Research in Electronics and Applied Physics, \\ University of Maryland, College Park, Maryland, USA; \\ ${ }^{c}$ Helmholtz-Zentrum Dresden-Rossendorf, Dresden, Germany; \\ ${ }^{d}$ Technische Universität Dresden, Dresden, Germany; \\ ${ }^{e}$ Department of Applied Physics, Chalmers University of Technology, Göteborg, Sweden
}

\begin{abstract}
We present an analytical expression for the differential transmission of a delta-shaped light field in Landauquantized graphene. This enables a direct comparison of experimental spectra to theoretical calculations reflecting the carrier dynamics including all relevant scattering channels. In particular, the relation is used to provide evidence for strong Auger scattering in Landau-quantized graphene.
\end{abstract}

Keywords: Landau-quantized graphene, Auger scattering, carrier dynamics, differential transmission spectroscopy

\section{INTRODUCTION}

Since its discovery in 2004, ${ }^{1}$ graphene has attracted an enormous amount of interest ${ }^{2}$ being considered as a novel material for optoelectronic devices. ${ }^{3-8}$ To exploit the fascinating electronic and optical properties of this atomically thin material, it is of crucial importance to understand the non-equilibrium carrier dynamics. There has been a lot of research in this field, ${ }^{9-27}$ revealing that carrier-carrier and carrier-phonon scattering dominate the ultrafast relaxation dynamics. ${ }^{28,29}$ Remarkably, Auger scattering - which is generally suppressed in ordinary semiconductors - turns out to be of particular importance in graphene, where it gives rise to carrier multiplication occurring at certain experimentally controllable conditions. ${ }^{23,30-33}$

In an external magnetic field, the electronic properties change dramatically, as the energy is quantized into non-equidistant Landau levels. ${ }^{34}$ Unlike in the case without a magnetic field, there are only a few studies of the carrier dynamics in Landau-quantized graphene. ${ }^{35-41}$ Here, optical selection rules ${ }^{42}$ allow the excitation of specific inter-Landau level transitions opening the possibility to address the carrier dynamics of selected levels in a pump-probe experiment. Plochocka et al. have investigated the carrier relaxation in highly energetic Landau levels (with an index $n \sim 100$ ) which was found to be slowed down by the magnetic field as a consequence of the energy quantization. ${ }^{35}$ In a more recent joint experiment theory study, we have investigated the carrier dynamics within the energetically lowest Landau levels. ${ }^{36}$ Based on a pump probe experiment and microscopic modeling of the carrier dynamics, we were able to provide evidence for strong Auger-type scattering in Landau-quantized graphene: Including this scattering channel in the theoretical model, an unexpected behavior in the measured differential transmission could be explained.

In this Article, we derive the analytic expression that was used in Ref. [36] to compare the experimental differential transmission spectra (DTS) with the calculations of the Landau level occupations. To this end, we introduce the Bloch equations for Landau-quantized graphene in Section 2, calculate the absorbance for a deltashaped probe pulse in Section 3, and ultimately derive an analytic expresion for the differential transmission in Section 4 .

\footnotetext{
*florian.wendler@tu-berlin.de, Telephone: +49 (0)30 31423029
} 


\section{BLOCH EQUATIONS FOR LANDAU-QUANTIZED GRAPHENE}

The many-particle Hamilton operator for charge carriers in Landau-quantized graphene subject to an optical light field is given by

$$
H=\sum_{i} \epsilon_{i} a_{i}^{\dagger} a_{i}+i \hbar \sum_{i, f} \Omega_{i f} a_{f}^{\dagger} a_{i}
$$

where the first term is the free energy part that is determined by the Landau level dispersion $\epsilon_{n}^{\lambda}=\lambda v_{\mathrm{F}} \sqrt{2 \hbar n e_{0} B}$ which depends on the band index $\lambda= \pm 1$, the Fermi velocity $v_{\mathrm{F}} \approx 1 \mathrm{~nm} / \mathrm{fs},{ }^{43}$ the Landau level index $n=$ $0,1,2, \ldots$, the elementary charge $e_{0}$, and the magnetic field $B$. The second term describes the carrier-light coupling expressed through the Rabi frequency $\Omega_{i f}(t)=\frac{e_{0}}{m_{0}} \mathbf{M}_{i f} \cdot \mathbf{A}(t)$ with the free electron mass $m_{0}$, the vector potential $\mathbf{A}(t)$, and the optical matrix element ${ }^{38,44}$

$$
\mathbf{M}_{i f}=i \delta_{\xi_{i}, \xi_{f}} \delta_{m_{i}, m_{f}} \frac{\alpha_{n_{i}} \alpha_{n_{f}} m_{0} v_{\mathrm{F}}}{2 \sqrt{2} \hbar}\left[\lambda_{i} \hat{\boldsymbol{\epsilon}}^{-} \delta_{n_{f}, n_{i}-1}+\lambda_{f} \hat{\boldsymbol{\epsilon}}^{+} \delta_{n_{f}, n_{i}+1}\right]=-\mathbf{M}_{f i}^{*}
$$

Here, $\xi= \pm 1$ is the valley index that distinguishes the two equivalent Dirac cones of graphene. ${ }^{34}$ Furthermore, $m=0,1, \ldots, L^{2} e_{0} B /(2 \pi \hbar)-1$ is a quantum number expressing the Landau level degeneracy with the area of graphene $L^{2},{ }^{34} \alpha_{n=0}$ is a constant that can take the values $\alpha_{n=0}=\sqrt{2}$ and $\alpha_{n \neq 0}=1$, and $\hat{\boldsymbol{\epsilon}}^{ \pm}=\left(\hat{\mathbf{e}}_{x} \mp i \hat{\mathbf{e}}_{y}\right) / \sqrt{2}$ are Jones vectors describing left $(+)$ and right $(-)$ circularly polarized light. ${ }^{45}$

The temporal evolution of the microscopic polarization $p_{i f}(t)=\left\langle a_{f}^{\dagger} a_{i}\right\rangle(t)$ (where $a_{j}^{\dagger}$ and $a_{j}$ are fermionic creation and annihilation operators of the state $j$ ) is obtained using the Heisenberg equation of motion $i \hbar \frac{\mathrm{d}}{\mathrm{d} t} \mathcal{O}(t)=$ $[\mathcal{O}, H]$ which provides an equation of motion for the operator $\mathcal{O} .{ }^{28,46}$ Using the Hamilton operator from Eq. 1, this yields

$$
\dot{p}_{i f}(t)=\left(i \triangle \omega_{i f}-\gamma\right) p_{i f}(t)-\Omega_{i f}^{*}\left[\rho_{f}(t)-\rho_{i}(t)\right],
$$

with the energy difference $\hbar \triangle \omega_{i f}=\epsilon_{f}-\epsilon_{i}$, a phenomenologically introduced dephasing $\gamma$, and the occupation probability $\rho_{j}=p_{j j}$ in the state $j$. The dephasing is equivalent to a Landau level broadening and can be caused by disorder ${ }^{47}$ or, alternatively by interaction with acoustic phonons. ${ }^{48,49}$

\section{ABSORBANCE OF A DELTA-SHAPED PULSE}

For a two-dimensional nanostructure in the linear optics regime, the absorbance reads ${ }^{26,28,29}$

$$
\alpha^{ \pm}(\omega)=\frac{\omega \operatorname{Im} \chi^{ \pm}(\omega)}{c\left|1+i \omega \chi^{ \pm}(\omega) / 2 c\right|^{2}}
$$

where $c$ is the light velocity and

$$
\chi^{ \pm}(\omega)=\frac{j^{ \pm}(\omega)}{\epsilon_{0} \omega^{2} A^{ \pm}(\omega)}
$$

is the linear optical susceptibility, with the current density $j$, and the permittivity $\epsilon_{0}$. The superscript \pm denotes the $\sigma^{ \pm}$-circularly polarized components of the respective quantity in the Jones vector basis $\left(\hat{\boldsymbol{\epsilon}}^{ \pm}\right)$. The components

$$
A^{ \pm}(\omega)=\frac{1}{2 \sqrt{\pi}}\left[A_{0}^{+} e^{i\left(\omega \mp \Delta \omega_{i f}\right) \tau}+A_{0}^{-} e^{i\left(\omega \pm \Delta \omega_{i f}\right) \tau}\right],
$$

of a $\delta$-shaped probe pulse that is in resonance with the transition $i \rightarrow f$ is obtained from

$$
\mathbf{A}(t)=\delta(t+\tau)\left[A_{0}^{+}\left(\begin{array}{c}
\cos \triangle \omega_{i f} t \\
\sin \triangle \omega_{i f} t
\end{array}\right)+A_{0}^{-}\left(\begin{array}{c}
\cos \triangle \omega_{i f} t \\
-\sin \triangle \omega_{i f} t
\end{array}\right)\right],
$$


where $\tau$ describes the temporal delay of the probe pulse, and $A_{0}^{ \pm}$are the amplitudes of the left $(+)$and right $(-)$ circularly polarized contributions. The current density is given by ${ }^{28}$

$$
j^{ \pm}(\omega)=-i \frac{\hbar e_{0}}{2 m_{0} L^{2}} \sum_{i f}\left[M_{i f}^{ \pm} p_{i f}(\omega)+M_{f i}^{ \pm} p_{f i}(\omega)\right] .
$$

where a term proportional to $A^{ \pm}$was neglected. Using the substitution $p_{i f}=p_{i f}^{\text {rot }} e^{i \triangle \omega_{i f} t}$, we transform Eq. 3 to the rotating frame

$$
\dot{p}_{i f}^{\mathrm{rot}}(t)=-\gamma p_{i f}^{\mathrm{rot}}(t)-\Omega_{i f}^{*}\left[\rho_{f}(t)-\rho_{i}(t)\right] e^{-i \triangle \omega_{i f} t},
$$

and obtain its Fourier transform (FT) exploiting the relation FT $\left[\dot{p}_{i f}^{\mathrm{rot}}(t)\right]=i \omega p_{i f}^{\mathrm{rot}}(\omega)$

$$
p_{i f}^{\mathrm{rot}}(\omega)=\delta_{\xi_{i}, \xi_{f}} \delta_{m_{i}, m_{f}} \frac{e_{0} v_{\mathrm{F}}}{4 \hbar} \alpha_{n_{i}} \alpha_{n_{f}}\left(\lambda_{i} \delta_{n_{f}, n_{i}-1} A_{0}^{-}+\lambda_{f} \delta_{n_{f}, n_{i}+1} A_{0}^{+}\right) \frac{\operatorname{FT}\left[\delta(t-\tau)\left(\rho_{f}-\rho_{i}\right)\right](\omega)}{\omega-i \gamma} .
$$

Here, the rotating wave approximation (RWA) was applied by omitting terms $e^{ \pm i 2 \Delta \omega_{i f} t}$ in comparison to 1 . Since the symmetry relation $p_{i f}^{*}=p_{f i}$ effects an inverse rotation of $p_{f i}$ compared to $p_{i f}$, cf. $p_{f i}=p_{f i}^{\text {rot }} e^{-i \triangle \omega_{i f} t}$, the RWA for $p_{f i}^{\text {rot }}(\omega)$ is not obtained by exchanging $i \leftrightarrow f$ in Eq. 10, but the relation $p_{f i}^{\text {rot }}(\omega)=-p_{i f}^{\text {rot }}(\omega)$ must be used instead. Exploiting the convolution theorem, we can write

$$
\begin{aligned}
\operatorname{FT}\left[\delta(t+\tau)\left(\rho_{f}-\rho_{i}\right)\right] & =\operatorname{FT}[\delta(t-\tau)] * \operatorname{FT}\left[\rho_{f}-\rho_{i}\right] \\
& =\frac{1}{2 \pi} e^{i \omega \tau}\left[\rho_{f}(\tau)-\rho_{i}(\tau)\right] .
\end{aligned}
$$

where $*$ denotes the convolution of two functions $f$ and $g:(f * g)(\omega)=\int_{-\infty}^{\infty} \mathrm{d} \omega^{\prime} f\left(\omega^{\prime}\right) g\left(\omega-\omega^{\prime}\right)$, Using Eqs. 2, 8, 10,11 , as well as the relations $p_{i f}(\omega)=p_{i f}^{\text {rot }}\left(\omega-\triangle \omega_{i f}\right)$ and $p_{f i}(\omega)=-p_{i f}^{\text {rot }}\left(\omega+\triangle \omega_{i f}\right)$, the current density reads

$$
j^{ \pm}(\omega)=\frac{e_{0}^{3} v_{\mathrm{F}}^{2} B}{64 \pi^{2} \hbar^{2}} \sum_{i f}\left(\alpha_{n_{i}} \alpha_{n_{f}}\right)^{2}\left[\rho_{f}(\tau)-\rho_{i}(\tau)\right]\left[A_{0}^{ \pm} \delta_{n_{f}, n_{i} \pm 1} \frac{e^{i\left(\omega-\Delta \omega_{i f}\right) \tau}}{\omega-\Delta \omega_{i f}-i \gamma_{i f}^{\mathrm{imp}}}-A_{0}^{\mp} \delta_{n_{f}, n_{i} \mp 1} \frac{e^{i\left(\omega+\Delta \omega_{i f}\right) \tau}}{\omega+\Delta \omega_{i f}-i \gamma_{i f}^{\mathrm{imp}}}\right]
$$

Here, we performed the summations over the valley and $m$ degrees of freedom. In due consideration of the Kronecker deltas $\delta_{\xi_{i}, \xi_{f}} \delta_{m_{i}, m_{f}}$ in Eq. 10 this yields a factor of $L^{2} e_{0} B /(\pi \hbar)$. Omitting terms with a non vanishing rotation $e^{i \tilde{\omega} \tau} \neq 1$, we obtain the linear optical susceptibility (Eq. 5) using the Eqs. 6 and 12

$$
\chi^{ \pm}(\omega)=\frac{\sqrt{\pi} e_{0}^{3} v_{\mathrm{F}}^{2} B}{32 \pi^{2} \hbar^{2} \epsilon_{0} \omega^{2}} \sum_{i f}\left(\alpha_{n_{i}} \alpha_{n_{f}}\right)^{2} \frac{\rho_{f}(\tau)-\rho_{i}(\tau)}{\omega-\Delta \omega_{i f}-i \gamma_{i f}^{\text {imp }}} \delta_{n_{f}, n_{i} \pm 1} .
$$

Neglecting the term $i \omega \chi(\omega) / 2 c$ in the denominator of Eq. 4 which is usually small, we find the final expression for the absorbance of a delta-shaped pulse

$$
\alpha^{ \pm}(\omega)=\frac{\sqrt{\pi} e_{0}^{3} v_{\mathrm{F}}^{2} B}{32 \pi^{2} \hbar^{2} \epsilon_{0} c \omega} \sum_{i f}\left(\alpha_{n_{i}} \alpha_{n_{f}}\right)^{2}\left[\rho_{f}(\tau)-\rho_{i}(\tau)\right] \frac{\gamma}{\left(\omega-\Delta \omega_{i f}\right)^{2}+\gamma^{2}} \delta_{n_{f}, n_{i} \pm 1}
$$

\section{DIFFERENTIAL TRANSMISSION}

The idea of the differential transmission spectroscopy is to optically excite the system under investigation using a strong pump pulse. Then, the differential transmission of a much weaker probe pulse incident after a certain time delay $\tau$ is measured. Based on the assumption that the occupations of the system are not affected by the weak probe pulse, the differential transmission allows to draw conclusions about the carrier dynamics in the system. Omitting possible interference between both pulses, which can lead to the emergence of a so-called coherent artifact, the ansatz

$$
\operatorname{DTS}(\omega, \tau)=\alpha_{\text {pump }}(\omega, \tau)-\alpha_{0}(\omega)
$$




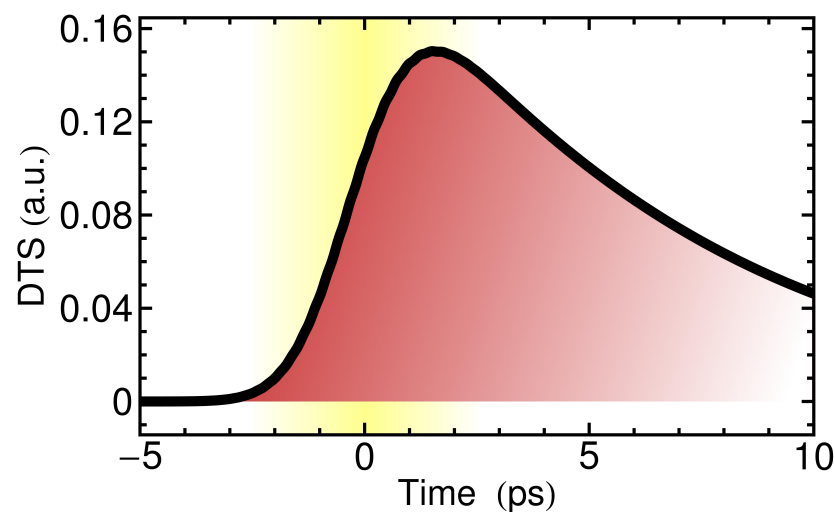

Figure 1. Differential transmission spectrum pumping and probing with linear polarized radiation and an energy matching the inter-Landau level transitions $\mathrm{LL}_{0} \rightarrow \mathrm{LL}_{+1}$ and $\mathrm{LL}_{-1} \rightarrow \mathrm{LL}_{0}$ at $B=4 \mathrm{~T}$. The yellow area in the background represents the pump pulse centered around $t=0$.

for the differential transmission is used, where $\alpha_{\text {pump }}\left(\alpha_{0}\right)$ is the absorbance of the probe pulse with (without) a previous pump pulse. Assuming a pump pulse centered around $t=0$, the initial absorbance of the probe pulse is given by $\alpha_{0}(\omega)=\alpha(\omega, \tau=-\infty)$, while the absorbance of the probe pulse following the pump pulse after a time $\tau$ is simply given by $\alpha_{\text {pump }}(\omega, \tau)=\alpha(\omega, \tau)$. Thereby, using Eq. 14, the DTS for a single transition $i \rightarrow f$ and a resonant excitation $\left(\omega=\Delta \omega_{i f}\right)$ reads

$$
\operatorname{DTS}_{i f}^{ \pm}(\tau)=\frac{\sqrt{\pi} e_{0}^{2} v_{\mathrm{F}}^{2}}{32 \pi^{2} \hbar \epsilon_{0} c l_{B}^{2} \Delta \omega_{i f} \gamma}\left(\alpha_{n_{i}} \alpha_{n_{f}}\right)^{2}\left[\Delta \rho_{f}(\tau)-\Delta \rho_{i}(\tau)\right] \delta_{n_{f}, n_{i} \pm 1},
$$

where $\Delta \rho_{j}(\tau)=\rho_{j}(\tau)-\rho_{j}(t=-\infty)$ is the occupation difference induced by the pump pulse. Note that (in RWA) $\sigma^{+}$-polarized radiation is only capable of exciting transitions where the Landau level index is incremented by one $n \rightarrow n+1$, while transitions with $n \rightarrow n-1$ require $\sigma^{-}$-polarized radiation (cf. $\delta_{n_{f}, n_{i} \pm 1}$ in Eq. 16, respectively). When more than one transition is addressed by the probe pulse at the same time, the DTS is given by the sum of the individual spectra (cf. sum over $i$ and $f$ in Eq. 14). Therefore, the DTS pumping and probing the inter-Landau level transitions $\mathrm{LL}_{0} \rightarrow \mathrm{LL}_{+1}$ and $\mathrm{LL}_{-1} \rightarrow \mathrm{LL}_{0}$ is given by $\operatorname{DTS}_{0 \rightarrow+1}^{+}(\tau)+\mathrm{DTS}_{-1 \rightarrow 0}^{-}(\tau)$ which is proportional to $\left(\Delta \rho_{+1}-\Delta \rho_{-1}\right)$. An example of such a calculated DTS for a linear polarized excitation of the energetically lowest Landau levels is shown in Fig. 1, where an increase during the optical excitation reflects the enhanced Pauli blocking induced by the pumping (absorption bleaching), and the decay results from the many-particle-induced relaxation back to the equilibrium distribution. Investigating the decay behavior (monoor multi-exponential) and the decay rate allows conclusions on the relaxation channels and their efficiency.

\section{CONCLUSION}

We have presented the derivation of the relation between the differential transmission accessible in pump-probe experiments and the occupation probabilities of Landau-quantized graphene. The obtained analytic expression shows that the differential transmission is proportional to the difference between the pump-induced occupation change in the final and the initial state.

\section{ACKNOWLEDGMENTS}

We acknowledge financial support from the Deutsche Forschungsgemeinschaft through SPP 1459. Furthermore, E. M. is thankful to the Swedish Research Council (VR). 


\section{REFERENCES}

[1] Novoselov, K. S., Geim, A. K., Morozov, S. V., Jiang, D., Zhang, Y., Dubonos, S. V., Grigorieva, I. V., and Firsov, A. A., "Electric Field Effect in Atomically Thin Carbon Films," Science 306, 666-669 (2004).

[2] Geim, A. K. and Novoselov, K. S., "The rise of graphene," Nat. Mater. 6(3), 183-191 (2007).

[3] Geim, A. K., "Graphene: Status and Prospects," Science 324, 1530-1534 (2009).

[4] Xia, F., Mueller, T., Lin, Y.-m., Valdes-Garcia, A., and Avouris, P., "Ultrafast graphene photodetector," Nature Nano. 4(12), 839-843 (2009).

[5] Bonaccorso, F., Sun, Z., Hasan, T., and Ferrari, A. C., "Graphene photonics and optoelectronics," Nature Photon. 4, 611-622 (2010).

[6] Sun, Z., Hasan, T., Torrisi, F., Popa, D., Privitera, G., Wang, F., Bonaccorso, F., Basko, D. M., and Ferrari, A. C., "Graphene Mode-Locked Ultrafast Laser," ACS Nano 4(2), 803-810 (2010).

[7] Avouris, P. and Dimitrakopoulos, C., "Graphene: synthesis and applications," Mater. Today 15(3), 86 - 97 (2012).

[8] Gan, X., Shiue, R.-J., Gao, Y., Meric, I., Heinz, T. F., Shepard, K., Hone, J., Assefa, S., and Englund, D., "Chip-integrated ultrafast graphene photodetector with high responsivity," Nature Photon. 7(11), 883-887 (2013).

[9] Kampfrath, T., Perfetti, L., Schapper, F., Frischkorn, C., and Wolf, M., "Strongly Coupled Optical Phonons in the Ultrafast Dynamics of the Electronic Energy and Current Relaxation in Graphite," Phys. Rev. Lett. 95, 187403 (2005).

[10] Dawlaty, J. M., Shivaraman, S., Chandrashekhar, M., Rana, F., and Spencer, M. G., "Measurement of ultrafast carrier dynamics in epitaxial graphene," Appl. Phys. Lett. 92, 042116 (2008).

[11] Sun, D., Wu, Z.-K., Divin, C., Li, X., Berger, C., de Heer, W. A., First, P. N., and Norris, T. B., "Ultrafast Relaxation of Excited Dirac Fermions in Epitaxial Graphene Using Optical Differential Transmission Spectroscopy," Phys. Rev. Lett. 101, 157402 (2008).

[12] Rana, F., George, P. A., Strait, J. H., Dawlaty, J., Shivaraman, S., Chandrashekhar, M., and Spencer, M. G., "Carrier recombination and generation rates for intravalley and intervalley phonon scattering in graphene," Phys. Rev. B 79, 115447 (2009).

[13] Winnerl, S., Orlita, M., Plochocka, P., Kossacki, P., Potemski, M., Winzer, T., Malic, E., Knorr, A., Sprinkle, M., Berger, C., de Heer, W. A., Schneider, H., and Helm, M., "Carrier Relaxation in Epitaxial Graphene Photoexcited Near the Dirac Point," Phys. Rev. Lett. 107, 237401 (2011).

[14] Breusing, M., Kuehn, S., Winzer, T., Malić, E., Milde, F., Severin, N., Rabe, J. P., Ropers, C., Knorr, A., and Elsaesser, T., "Ultrafast nonequilibrium carrier dynamics in a single graphene layer," Phys. Rev. B 83, 153410 (2011).

[15] Kim, R., Perebeinos, V., and Avouris, P., "Relaxation of optically excited carriers in graphene," Phys. Rev. $B$ 84, 075449 (2011).

[16] Winzer, T., Knorr, A., Mittendorff, M., Winnerl, S., Lien, M.-B., Sun, D., Norris, T. B., Helm, M., and Malic, E., "Absorption saturation in optically excited graphene," Appl. Phys. Lett. 101(22), 221115 (2012).

[17] Malic, E., Winzer, T., and Knorr, A., "Efficient orientational carrier relaxation in optically excited graphene," Appl. Phys. Lett. 101(21), 213110 (2012).

[18] Sun, D., Divin, C., Mihnev, M., Winzer, T., Malic, E., Knorr, A., Sipe, J. E., Berger, C., de Heer, W. A., First, P. N., and Norris, T. B., "Current relaxation due to hot carrier scattering in graphene," New J. Phys. 14(10), 105012 (2012).

[19] Gierz, I., Petersen, J. C., Mitrano, M., Cacho, C., Turcu, I. C. E., Springate, E., Stöhr, A., Köhler, A., Starke, U., and Cavalleri, A., "Snapshots of non-equilibrium Dirac carrier distributions in graphene," Nature Mater. 12(12), 1119-1124 (2013).

[20] Winnerl, S., Göttfert, F., Mittendorff, M., Schneider, H., Helm, M., Winzer, T., Malic, E., Knorr, A., Orlita, M., Potemski, M., Sprinkle, M., Berger, C., and de Heer, W. A., "Time-resolved spectroscopy on epitaxial graphene in the infrared spectral range: relaxation dynamics and saturation behavior," J. Phys. Condens. Matter 25(5), 054202 (2013).

[21] Johannsen, J. C., Ulstrup, S., Cilento, F., Crepaldi, A., Zacchigna, M., Cacho, C., Turcu, I. C. E., Springate, E., Fromm, F., Raidel, C., Seyller, T., Parmigiani, F., Grioni, M., and Hofmann, P., "Direct View of Hot Carrier Dynamics in Graphene," Phys. Rev. Lett. 111, 027403 (2013). 
[22] Winzer, T., Malić, E., and Knorr, A., "Microscopic mechanism for transient population inversion and optical gain in graphene," Phys. Rev. B 87, 165413 (2013).

[23] Plötzing, T., Winzer, T., Malic, E., Neumaier, D., Knorr, A., and Kurz, H., "Experimental Verification of Carrier Multiplication in Graphene," Nano Lett. 14(9), 5371-5375 (2014). PMID: 25144320.

[24] Mittendorff, M., Winzer, T., Malic, E., Knorr, A., Berger, C., de Heer, W. A., Schneider, H., Helm, M., and Winnerl, S., "Anisotropy of Excitation and Relaxation of Photogenerated Charge Carriers in Graphene," Nano Lett. 14(3), 1504-1507 (2014). PMID: 24559191.

[25] Jago, R., Winzer, T., Knorr, A., and Malic, E., "Graphene as Gain Medium for Broadband Lasers," arxiv:1409.8182 (2014).

[26] Knorr, A., Hughes, S., Stroucken, T., and Koch, S. W., "Theory of ultrafast spatio-temporal dynamics in semiconductor heterostructures ," Chemical Physics 210, 27 - 47 (1996). Confined Excitations in Molecular and Semiconductor Nanostructures.

[27] Winzer, T., Ciesielski, R., Handloser, M., Comin, A., Hartschuh, A., and Malic, E., "Microscopic view on the ultrafast photoluminescence from photo-excited graphene," arxiv:1411.0531 (2014).

[28] Malic, E. and Knorr, A., [Graphene and Carbon Nanotubes: Ultrafast Optics and Relaxation Dynamics], Wiley-VCH (2013).

[29] Malic, E., Winzer, T., Bobkin, E., and Knorr, A., "Microscopic theory of absorption and ultrafast manyparticle kinetics in graphene," Phys. Rev. B 84, 205406 (2011).

[30] Rana, F., "Electron-hole generation and recombination rates for Coulomb scattering in graphene," Phys. Rev. B 76, 155431 (2007).

[31] Winzer, T., Knorr, A., and Malic, E., "Carrier Multiplication in Graphene," Nano Lett. 10, 4839-4843 (2010).

[32] Winzer, T. and Malic, E., "Impact of Auger processes on carrier dynamics in graphene," Phys. Rev. B 85, 241404 (2012).

[33] Brida, D., Tomadin, A., Manzoni, C., Kim, Y. J., Lombardo, A., Milana, S., Nair, R. R., Novoselov, K. S., Ferrari, A. C., Cerullo, G., and Polini, M., "Ultrafast collinear scattering and carrier multiplication in graphene," Nature Commun. 4, 1987 (2013).

[34] Goerbig, M. O., "Electronic properties of graphene in a strong magnetic field," Rev. Mod. Phys. 83, 11931243 (2011).

[35] Plochocka, P., Kossacki, P., Golnik, A., Kazimierczuk, T., Berger, C., de Heer, W. A., and Potemski, M., "Slowing hot-carrier relaxation in graphene using a magnetic field," Phys. Rev. B 80, 245415 (2009).

[36] Mittendorff, M., Wendler, F., Malic, E., Knorr, A., Orlita, M., Potemski, M., Berger, C., de Heer, W. A., Schneider, H., Helm, M., and Winnerl, S., "Carrier dynamics in Landau-quantized graphene featuring strong Auger scattering," Nature Phys. 11, 75-81 (2015).

[37] Wendler, F., Knorr, A., and Malic, E., "Resonant carrier-phonon scattering in graphene under Landau quantization," Appl. Phys. Lett. 103(25), 253117 (2013).

[38] Wendler, F., Knorr, A., and Malic, E., "Carrier multiplication in graphene under Landau quantization," Nature Commun. 5, 3703 (2014).

[39] Wendler, F. and Malic, E., "Population inversion in Landau-quantized graphene," arXiv:1410.2080 (2014).

[40] Mittendorff, M., Orlita, M., Potemski, M., Berger, C., de Heer, W. A., Schneider, H., Helm, M., and Winnerl, S., "Intraband carrier dynamics in Landau-quantized multilayer epitaxial graphene," New J. Phys. 16, 123021 (2014).

[41] Wang, Z.-W., Liu, L., and Li, Z.-Q., "Fast two-phonon relaxation process between the Landau levels of graphene on different polar substrates," Europhys. Lett. 108, 36005 (2014).

[42] Rioux, J., Burkard, G., and Sipe, J. E., "Current injection by coherent one- and two-photon excitation in graphene and its bilayer," Phys. Rev. B 83, 195406 (2011).

[43] Castro Neto, A. H., Guinea, F., Peres, N. M. R., Novoselov, K. S., and Geim, A. K., "The electronic properties of graphene," Rev. Mod. Phys. 81, 109-162 (2009).

[44] Rao, K. M. and Sipe, J. E., "Coherent photocurrent control in graphene in a magnetic field," Phys. Rev. $B$ 86, 115427 (2012).

[45] Jones, R. C., "A New Calculus for the Treatment of Optical Systems," J. Opt. Soc. Am. 31, 488-493 (1941).

[46] Haug, H. and Koch, S. W., [Quantum Theory of the Optical and Electronic Properties of Semiconductors], World Scientific (2009). 
[47] Ando, T. and Uemura, Y., "Theory of Quantum Transport in a Two-Dimensional Electron System under Magnetic Fields. I. Characteristics of Level Broadening and Transport under Strong Fields," J. Phys. Soc. Jpn. 36, 959-967 (1974).

[48] Stock, E., Dachner, M.-R., Warming, T., Schliwa, A., Lochmann, A., Hoffmann, A., Toropov, A. I., Bakarov, A. K., Derebezov, I. A., Richter, M., Haisler, V. A., Knorr, A., and Bimberg, D., "Acoustic and optical phonon scattering in a single $\operatorname{In}(\mathrm{Ga})$ As quantum dot," Phys. Rev. B 83, 041304 (2011).

[49] Förstner, J., Weber, C., Danckwerts, J., and Knorr, A., "Phonon-induced damping of Rabi oscillations in semiconductor quantum dots," Phys. Status Solidi B 238, 419-422 (2003). 